\title{
Global Behaviors of a Chemostat Model with Delayed Nutrient Recycling and Periodically Pulsed Input
}

\author{
Kai Wang, ${ }^{1}$ Zhidong Teng, $^{1}$ and Fengqin Zhang ${ }^{2}$ \\ ${ }^{1}$ College of Mathematics and System Sciences, Xinjiang University, Urumqi 830046, China \\ 2 Department of Mathematics, Yuncheng University, Yuncheng 044000, China
}

Correspondence should be addressed to Fengqin Zhang, zhafq@263.net

Received 27 July 2010; Accepted 20 September 2010

Academic Editor: Antonia Vecchio

Copyright (c) 2010 Kai Wang et al. This is an open access article distributed under the Creative Commons Attribution License, which permits unrestricted use, distribution, and reproduction in any medium, provided the original work is properly cited.

The dynamic behaviors in a chemostat model with delayed nutrient recycling and periodically pulsed input are studied. By introducing new analysis technique, the sufficient and necessary conditions on the permanence and extinction of the microorganisms are obtained. Furthermore, by using the Liapunov function method, the sufficient condition on the global attractivity of the model is established. Finally, an example is given to demonstrate the effectiveness of the results in this paper.

\section{Introduction}

This paper is mainly concerned with single-species chemostat-type model with nutrient recycling. Usually, nutrient recycling is regarded as an instantaneous term by neglecting the time required to regenerate nutrient from dead biomass by bacterial decomposition. The motivation for such models is given by Beretta et al. in [1], where such systems are used to model the growth of planktonic communities in lakes, where the plankton feeds on a limiting nutrient supplied at a constant rate. The basic single-species chemostat model with delayed nutrient recycling is the following differential equation:

$$
\begin{gathered}
\frac{d S}{d t}=D\left(S^{0}-S\right)-m U(S) N+b D_{1} \int_{-\infty}^{t} F(t-\tau) N(\tau) d \tau, \\
\frac{d N}{d t}=N\left[-\left(D+D_{1}\right)+m_{1} U(S)\right] .
\end{gathered}
$$


The chemostat models with nutrient recycling have been extensively investigated by many researchers. The studied main subjects are the persistence, permanence, and extinction of microorganisms, global stability and the existence of periodic oscillation of the systems, and so forth. Many important and interesting results can be found in [1-15] and the references cited therein. In [4], Freedman and Xu extended the single-species model proposed in [1] to two-species competition models with instantaneous and delayed nutrient recycling. They developed persistence and extinction criteria for the competing populations. In [10], by applying the method of Liapunov functionals they study the global asymptotic stability of the positive equilibria of the models in [4]. In [12], a chemostat model with distributed time delays both in material recycling and biotic species growth has been considered.

As it is well known, countless organisms live in seasonally or diurnally forced environment, in which the populations obtain food, so the effects of this forcing may be quite profound. Recently many papers studied chemostat model with variations in the supply of nutrients or the washout. The chemostat models with impulsive input perturbation have been studied in many articles see [16-22] and the references cited therein, where many important and interesting results on the persistence, permanence and extinction of microorganisms, global stability, the existence of periodic oscillation and dynamical complexity of the systems are discussed. Particular in [21], the following model of the lactic acid fermentation in membrane bioreactor with impulsive input is discussed:

$$
\begin{aligned}
& \frac{d S}{d t}=\frac{Q}{V}\left(S^{0}-S\right)-\frac{\mu S x}{\delta_{1}\left(K_{S}+S\right)}+\left(\frac{K_{D}}{\delta_{1}}-m\right) x, \quad t \neq n T, \\
& \frac{d x}{d t}=\frac{\mu S x}{K_{S}+S}-\left(K_{D}+\frac{Q_{1}}{V}\right) x, \\
& \Delta S=\frac{Q S^{0}}{V}, \quad t=n T, n \in N . \\
& \Delta x=0 .
\end{aligned}
$$

The model has both nutrient recycling and impulsive input substrate. Using Floquet's theory of impulsive periodic linear differential equations and small-amplitude perturbation, they obtain the biomass-free periodic solution is locally stable if some conditions are satisfied, see [21, Theorems 3.1]. In [18], the following Monod type chemostat model with nutrient recycling and impulsive input is studied:

$$
\begin{aligned}
& \frac{d S(t)}{d t}=-D S(t)-\frac{\mu_{m} S(t) x(t)}{\delta\left(K_{m}+S(t)\right)}+b \gamma x(t), \quad t \neq n T, \\
& \frac{d x(t)}{d t}=\frac{\mu_{m} S(t) x(t)}{K_{m}+S(t)}-(D+\gamma) x(t), \\
& S\left(t^{+}\right)=S(t)+D S^{0}, \quad t=n T, n \in N . \\
& x\left(t^{+}\right)=x(t),
\end{aligned}
$$

The sufficient and necessary conditions of the permanence and extinction of the microorganism species and the sufficient condition of the global asymptotic stability of the model are established. 
However, we see that few authors consider the chemostat models with delayed nutrient recycling and periodically pulsed input. Based on the ideas given in [18], we develop model (1.1) into the following form by introducing impulsive input:

$$
\begin{aligned}
& \frac{d x(t)}{d t}=-d x-m U(x) y+b d_{1} \int_{-\infty}^{0} F(s) y(t+s) d s, \quad t \neq n T, \\
& \frac{d y(t)}{d t}=y\left[-\left(d+d_{1}\right)+m_{1} U(x)\right] \\
& x\left(t^{+}\right)=x(t)+p, \quad t=n T, n \in N . \\
& y\left(t^{+}\right)=y(t),
\end{aligned}
$$

For system (1.4), we will investigate the permanence, extinction and the global attractivity. We will establish the sufficient and necessary conditions for the permanence, extinction.

The rest of this paper is organized as follows. In the following section we will firstly introduce the basic assumption for system (1.4). Next, we will give a equivalent form of system (1.4) by introducing a new variable. Further, we will give several useful lemmas. In Section 3 we will state and prove a boundedness result for system (1.4). In Section 4 we will state and prove an extinction result and a permanence result for system (1.4). In Section 5 we will state and prove a global attractivity result for system (1.4). Finally, in Section 6, we will discuss an example and give some numerical simulations.

\section{Preliminaries}

In system (1.4), $x$ is the concentration of a limiting nutrient and $y$ is a measure of the population of some organism; $d>0$ is the input and output flow, and is referred to as the wash-out rate; $m>0$ is the maximum uptake rate of nutrient, $m_{1}>0$ is the maximum specific growth rate of the organism; $d_{1}>0$ is the death rate; $b \in(0,1)$ is the fraction of nutrient recycled after death of the species; $p>0$ is the amount of the substrate concentration pulsed each $n T$, where $T>0$ is a constant; $N$ represents the set of all positive integers; $U(s)$ is the uptake function; Delay-kernel function $F(u)$ is a nonnegative bounded integrable function defined on $R_{+}=[0, \infty)$. In this paper, we always assume that $d, m, d_{1}, m_{1}, b$ and $p$ are constants, and $F(u)=a e^{-a u}$, where $a>0$ is a constant.

For system (1.4), we always assume that uptake function $U(s)$ satisfies the following assumption:

(H) $U(0)=0, U(s)$ is continuously differentiable for all $s \geq 0$ and $d U(s) / d t>0$ for all $s \in\left[0, M_{0}\right]$, where $M_{0}=p /\left(1-e^{-d T}\right)$.

The initial conditions in system (1.4) are given in the following form:

$$
\begin{gathered}
x(0)=x_{0}>0, \\
y(\theta)=\varphi(\theta) \geq 0, \quad \theta \in R_{-}, \quad \varphi(0)>0, \quad \sup _{\theta \in R_{-}} \varphi(\theta)<\infty,
\end{gathered}
$$

where $R_{-}=(-\infty, 0]$. It is easy to prove that solution $(x(t), y(t))$ of system (1.4) with initial condition (2.1) is positive, that is, $x(t)>0$ and $y(t)>0$ in the interval of the existence. 
Now, for system (1.4) we introduce a new variable $z(t)$ as follows:

$$
z(t)=\int_{-\infty}^{t} F(t-\tau) y(\tau) d \tau
$$

Then, system (1.4) is equivalent to the following system:

$$
\begin{aligned}
& \frac{d x(t)}{d t}=-d x(t)-m U(x(t)) y(t)+b d_{1} z(t), \\
& \frac{d y(t)}{d t}=y(t)\left[-\left(d+d_{1}\right)+m_{1} U(x(t))\right], \quad t \neq n T, \\
& \frac{d z(t)}{d t}=-a z(t)+a y(t), \\
& x\left(t^{+}\right)=x(t)+p, \\
& y\left(t^{+}\right)=y(t), \quad t=n T, n \in N, \\
& z\left(t^{+}\right)=z(t),
\end{aligned}
$$

where variable $z(t)$ can be interpreted as an intermediate component. Besides, initial condition (2.1) is changed into the following form

$$
x(0)=x_{0}>0, \quad y(0)=y_{0}>0, \quad z(0)=z_{0}>0 .
$$

Therefore, in the rest of this paper we will mainly discuss system (2.3).

Firstly, on the positivity of solutions for system (2.3), we have the following result.

Lemma 2.1. The solution $(x(t), y(t), z(t))$ of system (2.3) with initial condition (2.4) is positive, that is, $x(t)>0, y(t)>0$ and $z(t)>0$ for any $t \geq 0$.

The proof of Lemma 2.1 is simple, we hence omit it here.

We consider the following linear impulsive differential equation:

$$
\begin{gathered}
\frac{d \omega(t)}{d t}=-d \omega(t)-\eta, \quad t \neq n T, \\
\omega\left(t^{+}\right)=\omega(t)+p, \quad t=n T, n \in N, \\
\omega\left(0^{+}\right)=\omega_{0},
\end{gathered}
$$

where $\eta$ is a constant. Clearly,

$$
\omega^{*}(t)=-\frac{\eta}{d}+x^{*}(t), \quad t \in(n T,(n+1) T], n \in N
$$


is the $T$-periodic solution of system (2.5), where

$$
x^{*}(t)=\frac{p e^{-d(t-n T)}}{1-e^{-d T}}, \quad t \in(n T,(n+1) T], n \in N
$$

We say that $\omega^{*}(t)$ is globally uniformly attractive, if for any constants $M>0$ and $\varepsilon>0$ there is a constant $T(M, \varepsilon)>0$ such that for any initial time $t_{0} \geq 0$ and any solution $\omega(t)$ of system (2.5) with $\left|w\left(t_{0}\right)\right| \leq M$, one has

$$
\left|w(t)-w^{*}(t)\right|<\varepsilon \quad \forall t \geq t_{0}+T(M, \varepsilon)
$$

We have the following result.

Lemma 2.2. T-periodic solution $\omega^{*}(t)$ of system (2.5) is globally uniformly attractive.

Proof. Let $\omega(t)$ be any solution of system (2.5) with initial value $\omega\left(t_{0}\right)$. Define $u(t)=\omega(t)-$ $\omega^{*}(t)$, then we have

$$
\begin{gathered}
\frac{d u(t)}{d t}=-d u(t), \quad t \neq n T, \\
u\left(t^{+}\right)=u(t), \quad t=n T, n \in N, \\
u\left(t_{0}\right)=\omega\left(t_{0}\right)-\omega^{*}\left(t_{0}\right) .
\end{gathered}
$$

The solution of system (2.9) is $u(t)=u\left(t_{0}\right) e^{-d\left(t-t_{0}\right)}, t \geq t_{0}$. For any constant $M>0$ and $\varepsilon>0$, when $\left|\omega\left(t_{0}\right)\right| \leq M$, we have

$$
\begin{aligned}
|u(t)| & =\left|u\left(t_{0}\right)\right| e^{-d\left(t-t_{0}\right)} \\
& \leq\left(M+M^{*}\right) e^{-d\left(t-t_{0}\right)}, \quad t \geq t_{0}
\end{aligned}
$$

where $M^{*}=\eta / d+p /\left(1-e^{-d T}\right)$. Choose $T(M, \varepsilon)=-(1 / d) \ln \left(\varepsilon /\left(M+M^{*}\right)\right)$; then for any $t_{0} \geq 0$ we can obtain

$$
|u(t)| \leq\left(M+M^{*}\right) e^{-d\left(t-t_{0}\right)} \leq 0
$$

for all $t \geq t_{0}+T(M, \varepsilon)$. This shows that solution $\omega^{*}(t)$ is globally uniformly attractive. This completes the proof.

In system (2.5), when $\eta=0$, then we obtain the subsystem of system (2.3) with $y(t)=0$ and $z(t)=0$ as follows:

$$
\begin{gathered}
\frac{d x(t)}{d t}=-d x(t), \quad t \neq n T, \\
x\left(t^{+}\right)=x(t)+p, \quad t=n T, n \in N .
\end{gathered}
$$


Clearly, $x^{*}(t)$ is the positive $T$-periodic solution of system (2.12). Therefore, system (2.3) has the semitrivial $T$-periodic solution $\left(x^{*}(t), 0,0\right)$. The solution of system (2.12) with initial condition $x\left(0^{+}\right)=x_{0}$ is

$$
x(t)=\left(x_{0}-\frac{p}{1-e^{-d T}}\right) e^{-d t}+x^{*}(t), \quad t \in(n T,(n+1) T], n \in N
$$

From Lemma 2.2, we obtain that for any solution $x(t)$ of system (2.12), one has

$$
\left|x(t)-x^{*}(t)\right| \longrightarrow 0 \quad \text { as } t \longrightarrow \infty
$$

\section{Boundedness}

On the ultimate boundedness of all positive solutions of system (2.3) we have the following result.

Theorem 3.1. Let $(x(t), y(t), z(t))$ be any positive solution of system (2.3) if

$$
0<\frac{b}{a-d}<\frac{m}{a m_{1}}
$$

then

$$
\limsup _{t \rightarrow \infty} x(t)<M_{0}, \quad \limsup _{t \rightarrow \infty} y(t)<\frac{m_{1}}{m} M_{0}, \quad \limsup _{t \rightarrow \infty} z(t)<\frac{(a-d) M_{0}}{b d_{1}},
$$

where $M_{0}=p /\left(1-e^{-d T}\right)$.

Proof. From condition (3.1), there is a constant $c>0$ such that

$$
b d_{1}+c d-a c<0, \quad a c-\frac{m d_{1}}{m_{1}}<0
$$

Let $(x(t), y(t), z(t))$ be any solution of system (2.3) define Liapunov function $V(t)$ as follows:

$$
V(t)=x(t)+\frac{m}{m_{1}} y(t)+c z(t)
$$

Calculating the derivative of $V(t)$ along solution $(x(t), y(t), z(t))$ of system (2.3), we have

$$
\begin{aligned}
\frac{d V(t)}{d t} & =\frac{d x(t)}{d t}+\frac{m}{m_{1}} \frac{d y(t)}{d t}+c \frac{d z(t)}{d t} \\
& =-d V(t)+\left(a c-\frac{m d_{1}}{m_{1}}\right) y+\left(b d_{1}+c d-a c\right) z \\
& \leq-d V(t)
\end{aligned}
$$


for all $t \geq 0$ and $t \neq n T$, and

$$
V\left(t^{+}\right)=V(t)+p, \quad t=n T, n \in N
$$

From the comparison theorem of impulse differential equations, we have

$$
V(t) \leq u(t), \quad \forall t \geq 0,
$$

where $u(t)$ is the solution of system (2.12) with initial value $u\left(0^{+}\right)=V\left(0^{+}\right)$. From Lemma 2.2, we have $u(t) \rightarrow x^{*}(t)$ as $t \rightarrow \infty$. Hence, we further obtain

$$
\limsup _{t \rightarrow \infty} V(t) \leq \frac{p}{1-e^{-d T}}=M_{0}
$$

From this, we finally obtain

$$
\limsup _{t \rightarrow \infty} x(t)<M_{0}, \quad \quad \limsup _{t \rightarrow \infty} y(t)<\frac{m_{1}}{m} M_{0}, \quad \limsup _{t \rightarrow \infty} z(t)<\frac{(a-d) M_{0}}{b d_{1}} .
$$

This completes the proof of Theorem 3.1.

Return to the original system (1.4), we have the following corollary as a consequence of Theorem 3.1.

Corollary 3.2. Let $(x(t), y(t))$ be any positive solution of system (1.4) if inequality (3.1) holds, then

$$
\limsup _{t \rightarrow \infty} x(t)<M_{0}, \quad \limsup _{t \rightarrow \infty} y(t)<\frac{m_{1}}{m} M_{0}
$$

where $M_{0}=p /\left(1-e^{-d T}\right)$.

Remark 3.3. Compare Theorem 3.1 with Lemma 2.3 given in [18] that we can see the ultimate boundedness is quite different between model with delayed nutrient recycling and the model with instantaneous nutrient recycling.

\section{Extinction and Permanence}

On the extinction of the microorganism species of system (2.3), we have the following result.

Theorem 4.1. Suppose that inequality (3.1) holds and

$$
\int_{0}^{T}\left[-\left(d+d_{1}\right)+m_{1} U\left(x^{*}(t)\right)\right] d t \leq 0
$$

Then periodic solution $\left(x^{*}(t), 0,0\right)$ of system (2.3) is globally attractive. 
Proof. From condition (3.1), there is a constant $c>0$ such that

$$
b d_{1}+c d-a c<0, \quad a c-\frac{m d_{1}}{m_{1}}<0
$$

Let $(x(t), y(t), z(t))$ be any solution of system (2.3), define Liapunov function $V(t)$ as follows

$$
V(t)=x(t)+\frac{m}{m_{1}} y(t)+c z(t)
$$

Then similar to the proof of Theorem 3.1. We obtain $V(t) \leq u(t)$ for all $t \geq 0$, where $u(t)$ is the solution of system (2.12) with initial value $u\left(0^{+}\right)=V\left(0^{+}\right)$and $u(t) \rightarrow x^{*}(t)$ as $t \rightarrow \infty$. Hence, there exists a function $\alpha(t): R_{+} \rightarrow R$ satisfying $\alpha(t) \rightarrow 0$ as $t \rightarrow \infty$ such that

$$
V(t) \leq u(t)=x^{*}(t)+\alpha(t)
$$

for all $t \geq 0$. From the definition of $V(t)$, we further have

$$
x(t) \leq x^{*}(t)+\alpha(t)-\frac{m}{m_{1}} y(t)-c z(t) .
$$

From the second equation of system (2.3), we obtain

$$
\frac{d y(t)}{d t} \leq y(t)\left[-\left(d+d_{1}\right)+m_{1} U\left(x^{*}(t)+\alpha(t)-\frac{m}{m_{1}} y(t)-c z(t)\right)\right]
$$

From condition (4.1), we obtain for any $\varepsilon_{0}>0$

$$
\int_{0}^{T}\left[-\left(d+d_{1}\right)+m_{1} U\left(x^{*}(t)-\frac{m}{m_{1}} \varepsilon_{0}\right)\right] d t<0 .
$$

Since $\lim _{t \rightarrow \infty} \alpha(t)=0$, we can obtain

$$
\begin{gathered}
\limsup _{t \rightarrow \infty} \int_{t}^{t+T}\left[-\left(d+d_{1}\right)+m_{1} U\left(x^{*}(t)+\alpha(t)-\frac{m}{m_{1}} \varepsilon_{0}-c z(t)\right)\right] d t \\
\quad \leq \lim _{t \rightarrow \infty} \int_{t}^{t+T}\left[-\left(d+d_{1}\right)+m_{1} U\left(x^{*}(t)+\alpha(t)-\frac{m}{m_{1}} \varepsilon_{0}\right)\right] d t \\
\quad=\int_{0}^{T}\left[-\left(d+d_{1}\right)+m p\left(s^{*}(t)-\frac{1}{m} \varepsilon_{0}\right)\right] d t<0 .
\end{gathered}
$$


Hence, there exist constants $\eta>0$ and $T_{0}>0$ such that when $t \geq T_{0}$,

$$
\int_{t}^{t+T}\left[-\left(d+d_{1}\right)+m p\left(s^{*}(t)+\alpha(t)-\frac{m}{m_{1}} \varepsilon_{0}-c z(t)\right)\right] \leq-\eta
$$

and $|\alpha(t)|<1$.

If $y(t) \geq \varepsilon_{0}$ for all $t \geq T_{0}$, then from (4.6) we obtain

$$
\frac{d y(t)}{d t} \leq y(t)\left[-\left(d+d_{1}\right)+m_{1} U\left(x^{*}(t)+\alpha(t)-\frac{m}{m_{1}} \varepsilon_{0}-c z(t)\right)\right]
$$

For any $t \geq T_{0}$, we choose an integer $p \geq 0$ such that $t \in\left(T_{0}+p T, T_{0}+(p+1) T\right]$; then integrating (4.10) from $T_{0}$ to $t$, from (4.9), we can obtain

$$
\begin{aligned}
y(t) & \leq y\left(T_{0}\right) \exp \left\{\int_{T_{0}}^{t}\left[-\left(d+d_{1}\right)+m_{1} U\left(x^{*}(t)+\alpha(t)-\frac{m}{m_{1}} \varepsilon_{0}-c z(t)\right)\right] d t\right\} \\
& \leq y\left(T_{0}\right) \exp (-\eta p) \exp \left\{\int_{T_{0}+p T}^{t}\left[-\left(d+d_{1}\right)+m_{1} U\left(x^{*}(t)+\alpha(t)-\frac{m}{m_{1}} \varepsilon_{0}-c z(t)\right)\right] d t\right\} \\
& \leq y\left(T_{0}\right) \exp (-\eta p) \exp \left(\left[U\left(M_{0}+1-\frac{m}{m_{1}} \varepsilon_{0}\right)-\left(d+d_{1}\right)\right] T\right)
\end{aligned}
$$

where constant $M_{0}$ is given in Theorem 3.1. Since $p \rightarrow \infty$ as $t \rightarrow \infty$, from (4.11), we obtain $y(t) \rightarrow 0$ as $t \rightarrow \infty$ which leads to a contradiction. Hence, there is a $t^{*} \geq T_{0}$ such that $y\left(t^{*}\right)<\varepsilon_{0}$.

Now, we claim that there exists a constant $M_{1}>1$ such that $y(t) \leq \varepsilon_{0} M_{1}$ for all $t \geq t^{*}$. In fact, if there exists a $t_{1}>t^{*}$ such that $y\left(t_{1}\right)>\varepsilon_{0} M_{1}$, then there exists a $t_{2} \in\left(t^{*}, t_{1}\right)$ such that $y\left(t_{2}\right)=\varepsilon_{0}$ and $y(t)>\varepsilon_{0}$ for $t \in\left(t_{2}, t_{1}\right)$. Choose an integer $p \geq 0$ such that $t_{1} \in\left[t_{2}+p T, t_{2}+(p+\right.$ $1) T)$. Since for any $t \in\left(t_{2}, t_{1}\right)$

$$
\frac{d y(t)}{d t} \leq y(t)\left[-\left(d+d_{1}\right)+m_{1} U\left(x^{*}(t)+\alpha(t)-\frac{m}{m_{1}} \varepsilon_{0}-c z(t)\right)\right]
$$

integrating this inequality from $t_{2}$ to $t_{1}$, from (4.9) we can obtain

$$
\begin{aligned}
y\left(t_{1}\right) & \leq y\left(t_{2}\right) \exp \left\{\int_{t_{2}}^{t_{1}}\left[-\left(d+d_{1}\right)+m_{1} U\left(x^{*}(t)+\alpha(t)-\frac{m}{m_{1}} \varepsilon_{0}-c z(t)\right)\right] d t\right\} \\
& \leq y\left(t_{2}\right) \exp (-\eta p) \exp \left\{\int_{t_{2}+p T}^{t_{1}}\left[-\left(d+d_{1}\right)+m_{1} U\left(x^{*}(t)+\alpha(t)-\frac{m}{m_{1}} \varepsilon_{0}\right)\right] d t\right\} \\
& \leq \varepsilon_{0} \exp \left(\left[U\left(M_{0}+1-\frac{m}{m_{1}} \varepsilon_{0}\right)-\left(d+d_{1}\right)\right] T\right) .
\end{aligned}
$$


Obviously, choose constant

$$
M_{1}=\exp \left(\left[U\left(M_{0}+1-\frac{m}{m_{1}} \varepsilon_{0}\right)-\left(d+d_{1}\right)\right] T\right)
$$

then from (4.13) we obtain a contradiction. Hence, we have $y(t) \leq \varepsilon_{0} M_{1}$ for all $t \geq t^{*}$. Since $\varepsilon_{0}$ is arbitrary, we finally have $\lim _{t \rightarrow \infty} y(t)=0$. Obviously, we can obtain from the third equation of system (2.3) $\lim _{t \rightarrow \infty} z(t)=0$ and further from the first equation of system (2.3) we can obtain easily that $\lim _{t \rightarrow \infty}\left(x(t)-x^{*}(t)\right)=0$. This completes the proof of Theorem 4.1.

On the permanence of the nutrient and the microorganism for system (2.3), we have the following result.

Theorem 4.2. Suppose that inequality (3.1) holds and

$$
\int_{0}^{T}\left[-\left(d+d_{1}\right)+m_{1} U\left(x^{*}(t)\right)\right] d t>0
$$

where $x^{*}(t)$ is the unique positive T-periodic solution of system (2.12). Then system (2.3) is permanent.

Proof. Let $(x(t), y(t), z(t))$ be any solution of system (2.3) with initial value (2.4). Since inequality (3.1) holds, from Theorem 3.1, for any $\varepsilon>0$ there is a $T_{1}>0$ such that

$$
x(t) \leq M_{0}+\varepsilon, \quad y(t) \leq \frac{m_{1}}{m}\left(M_{0}+\varepsilon\right), \quad \forall t \geq T_{1} .
$$

From assumption $(\mathbf{H})$ and the theorem of mean value, for all $t \geq 0$, there exists a $\xi(t) \in(0, x(t))$ such that $U(x(t))=(d U(\xi(t)) / d s) x(t)$. Since $d U(s) / d s$ is continuous for $s \geq 0$, there exists a constant $M_{1}>0$ such that

$$
\frac{U(x(t))}{x(t)}=\frac{d U(\xi(t))}{d s} \leq M_{1}, \quad \forall t \geq 0 .
$$

From the first equation of system (2.3), we obtain

$$
\begin{aligned}
\frac{d x(t)}{d t} & \geq-d x(t)-m U(x(t)) y(t) \\
& \geq-d x(t)-m_{1}\left(M_{0}+\varepsilon\right) \frac{d U(\xi(t))}{d s} x(t) \\
& \geq-\left(d+m_{1}\left(M_{0}+\varepsilon\right) M_{1}\right) x(t)
\end{aligned}
$$


for all $t \geq T_{1}$ and $t \neq n T$, and $x\left(t^{+}\right)=x(t)+p, t=n T, n \in N$. Using the comparison theorem of impulsive differential equation, we obtain $x(t) \geq v(t)$ for all $t \geq T_{1}$, where $v(t)$ is the solution of the following impulsive equation:

$$
\begin{gathered}
\frac{d v(t)}{d t}=-\left(d+m_{1}\left(M_{0}+\varepsilon\right) M_{1}\right) v(t), \quad t \neq n T, \\
v\left(t^{+}\right)=v(t)+p, \quad t=n T, n \in N,
\end{gathered}
$$

with initial condition $v\left(T_{1}^{+}\right)=x\left(T_{1}^{+}\right)$. Further from Lemma 2.2, we have

$$
\lim _{t \rightarrow \infty}\left(v(t)-v^{*}(t)\right)=0,
$$

where $v^{*}(t)$ is the unique $T$-periodic solution of (4.19) and

$$
v^{*}(t)=\frac{p \exp \left\{-\left(d+m_{1}\left(M_{0}+\varepsilon\right) M_{1}\right)(t-n T)\right\}}{1-\exp \left\{-\left(d+m_{1}\left(M_{0}+\varepsilon\right) M_{1}\right) T\right\}}
$$

for all $t \in(n T,(n+1) T]$ and $n \in N$. Therefore, we further obtain

$$
\liminf _{t \rightarrow \infty} x(t) \geq \liminf _{t \rightarrow \infty} v(t) \geq \frac{p \exp \left(-\left(d+m_{1}\left(M_{0}+\varepsilon\right) M_{1}\right) T\right)}{1-\exp \left(-\left(d+m_{1}\left(M_{0}+\varepsilon\right) M_{1}\right) T\right)}
$$

This shows that $x(t)$ in system (2.3) is permanent.

Next, we prove that there exists a constant $m_{2}>0$ such that $\liminf _{t \rightarrow \infty} y(t) \geq m_{2}$ for any solution $(x(t), y(t), z(t))$ of system (2.3) with initial value (2.4). From assumption $(\mathbf{H})$, we can choose a constant $\varepsilon_{0}$ such that $d U(s) / d s>0$ for all $s \in\left[0, M_{0}+\varepsilon_{0}\right]$. According to (4.6), we can choose positive constants $\varepsilon$, $\varepsilon_{1}$ and $\varepsilon_{2}$, and $\varepsilon \leq \varepsilon_{0}$, such that

$$
\int_{0}^{T}\left[-\left(d+d_{1}\right)+m_{1} U\left(x^{*}(t)-\frac{m U\left(M_{0}+\varepsilon\right)}{d} \varepsilon_{1}-\varepsilon_{2}\right)\right] d t>\varepsilon_{2}
$$

We first prove that there is a constant $\eta>0$ and $\eta<\varepsilon_{1}$ such that

$$
\limsup _{t \rightarrow \infty} y(t) \geq \eta
$$

for any solution $(x(t), y(t), z(t))$ of system (2.3) with initial value (2.4).

In fact, if (4.24) is not true, then there is a solution $(x(t), y(t), z(t))$ of system (2.3) such that $\limsup _{t \rightarrow \infty} y(t) \leq \varepsilon_{1}$. Hence, there is a $T_{0}>0$ such that $y(t)<\varepsilon_{1}$, for all $t \geq T_{0}$. Further, from Theorem 3.1, there exists a constant $T_{1} \geq T_{0}$ such that for all $t \geq T_{1}$

$$
x(t)<M_{0}+\varepsilon, \quad y(t)<\frac{m_{1}}{m}\left(M_{0}+\varepsilon\right) .
$$


From the first equation of system (2.3), we have

$$
\begin{aligned}
\frac{d x(t)}{d t} & \geq-d x(t)-m U(x(t)) y(t) \\
& \geq-d x(t)-\varepsilon_{1} m U\left(M_{0}+\varepsilon\right), \quad t \geq T_{1}, \quad t \neq n T, \\
x\left(t^{+}\right) & =x(t)+p, \quad t=n T, n \in N .
\end{aligned}
$$

From the comparison theorem of impulse differential equations and Lemma 2.2, for above $\varepsilon_{2}>0$, there is a $n_{1} \in N$ and $n_{1} T>T_{1}$ such that

$$
x(t) \geq-\frac{m U\left(M_{0}+\varepsilon\right)}{d} \varepsilon_{1}+x^{*}(t)-\varepsilon_{2}
$$

for all $t \geq n_{1} T$. Then from the second equation of system (2.3), we have that

$$
\frac{d y(t)}{d t} \geq y(t)\left[-\left(d+d_{1}\right)+m_{1} U\left(x^{*}(t)-\frac{m U\left(M_{0}+\varepsilon\right)}{d} \varepsilon_{1}-\varepsilon_{2}\right)\right]
$$

for all $t>n_{1} T$. Integrating (4.29) from $n_{1} T$ to $t \geq n_{1} T$, we obtain

$$
\frac{d y(t)}{d t} \geq y\left(n_{1} T\right) \exp \int_{n_{1} T}^{t}\left[-\left(d+d_{1}\right)+m_{1} U\left(x^{*}(t)-\frac{m U\left(M_{0}+\varepsilon\right)}{d} \varepsilon_{1}-\varepsilon_{2}\right)\right] d t .
$$

Obviously, from (4.23) and (4.29), we obtain $y(t) \rightarrow \infty$ as $t \rightarrow \infty$, which leads to a contradiction. Therefore, (4.24) is true.

Now, we prove $y(t)$ in system (2.3) is permanent. Assume that it is not true, then there exists a sequence of initial values $\left\{\varphi_{k}\right\}=\left\{\left(x_{0}^{(k)}, y_{0}^{(k)}, z_{0}^{(k)}\right)\right\}$ which satisfies initial condition (2.4) such that for solution $\left(x\left(t, \varphi_{k}\right), y\left(t, \varphi_{k}\right), z\left(t, \varphi_{k}\right)\right)$, of system (2.3),

$$
\liminf _{t \rightarrow \infty} y\left(t, \varphi_{k}\right)<\frac{\eta}{k^{2}}, \quad \forall k=1,2, \ldots
$$

From (4.24) and (4.30) we obtain that there exist two time sequences $\left\{s_{q}^{(k)}\right\}$ and $\left\{t_{q}^{(k)}\right\}$ such that for each $k=1,2, \ldots$,

$$
\begin{gathered}
0<s_{1}^{(k)}<t_{1}^{(k)}<s_{2}^{(k)}<t_{2}^{(k)}<\cdots<s_{q}^{(k)}<t_{q}^{(k)}<\cdots, \\
s_{q}^{(k)} \longrightarrow \infty, \quad t_{q}^{(k)} \longrightarrow \infty \quad \text { as } q \longrightarrow \infty \\
y\left(t_{q}^{(k)}, \varphi_{k}\right)=\frac{\eta}{k^{2}}, \quad y\left(s_{q}^{(k)}, \varphi_{k}\right)=\frac{\eta}{k^{\prime}} \\
\frac{\eta}{k^{2}}<y\left(t, \varphi_{k}\right)<\frac{\eta}{k} \quad \forall t \in\left(s_{q}^{(k)}, t_{q}^{(k)}\right) .
\end{gathered}
$$


From Theorem 3.1, there is a $T^{(k)} \geq T_{0}$ such that

$$
y\left(t, \varphi_{k}\right) \leq \frac{m_{1}}{m}\left(M_{0}+\varepsilon\right), \quad x\left(t, \varphi_{k}\right) \leq M_{0}+\varepsilon \quad \forall t \geq T^{(k)} .
$$

Further, from (4.31) for every $k$ there is an integer $K^{(k)}>0$ such that $s_{q}^{(k)}>T^{(k)}$ for all $q \geq K^{(k)}$. Hence, for any $t \in\left[s_{q}^{(k)}, t_{q}^{(k)}\right]$ and $q \geq K^{(k)}$, we have

$$
\begin{aligned}
\frac{d y\left(t, \varphi_{k}\right)}{d t} & =y\left(t, \varphi_{k}\right)\left[-\left(d+d_{1}\right)+m_{1} U\left(x\left(t, \varphi_{k}\right)\right)\right] \\
& \geq-\gamma y\left(t, \varphi_{k}\right)
\end{aligned}
$$

where $\gamma=d+d_{1}+m_{1} U\left(M_{0}+\varepsilon\right)$. Therefore for any $q \geq K^{(k)}$ and $k=1,2, \ldots$, integrating (4.35) on $\left[s_{q}^{(k)}, t_{q}^{(k)}\right]$, we obtain from $(4.32)$

$$
\begin{aligned}
\frac{\eta}{k^{2}} & =y\left(t_{q}^{(k)}, \varphi_{k}\right) \\
& \geq y\left(s_{q}^{(k)}, \varphi_{k}\right) \exp \left[-\gamma\left(t_{q}^{(k)}-s_{q}^{(k)}\right)\right] \\
& =\frac{\eta}{k} \exp \left[-\gamma\left(t_{q}^{(k)}-s_{q}^{(k)}\right)\right] .
\end{aligned}
$$

Consequently,

$$
t_{q}^{(k)}-s_{q}^{(k)} \geq \frac{\ln k}{\gamma} \quad \forall q \geq K^{(k)}, k=1,2, \ldots
$$

Since for any integer $k, q \geq K^{(k)}, t \in\left[s_{q}^{(k)}, t_{q}^{(k)}\right]$ and $q \geq K^{(k)}$ we have

$$
\begin{aligned}
\frac{d x\left(t, \varphi_{k}\right)}{d t} & =-d x\left(t, \varphi_{k}\right)-m y\left(t, \varphi_{k}\right) U\left(s\left(t, \varphi_{k}\right)\right)+b d_{1} z\left(t, \varphi_{k}\right) \\
& \geq-d x\left(t, \varphi_{k}\right)-m y\left(t, \varphi_{k}\right) U\left(x\left(t, \varphi_{k}\right)\right) \\
& \geq-d x\left(t, \varphi_{k}\right)-m \varepsilon_{1} U\left(M_{0}+\varepsilon\right) .
\end{aligned}
$$

By the comparison theorem it follows that $x\left(t, \varphi_{k}\right) \geq \bar{x}(t)$, forall $t \in\left[s_{q}^{(k)}, t_{q}^{(k)}\right]$, where $\bar{x}(t)$ is the solution of the following system with initial condition $\bar{x}\left(s_{q}^{(k)}\right)=x\left(s_{q}^{(k)}, \varphi_{k}\right)$ :

$$
\begin{gathered}
\frac{d \omega(t)}{d t}=-d \omega(t)-m \varepsilon_{1} U\left(M_{0}+\varepsilon\right), \quad t \neq n T, \\
\omega\left(t^{+}\right)=\omega(t)+p, \quad t=n T, n \in N .
\end{gathered}
$$


By Lemma 2.2, system (4.39) has a unique positive $T$-periodic solution

$$
\omega^{*}(t)=x^{*}(t)-\frac{m U\left(M_{0}+\varepsilon\right)}{d} \varepsilon_{1}
$$

and $\omega^{*}(t)$ is globally uniformly attractive. Hence, for above $\varepsilon_{2}$, there is a constant $T^{*}>0$, and $T^{*}$ is independent of any $n$ and $q \geq K^{(k)}$, such that

$$
\bar{x}(t) \geq x^{*}(t)-\frac{m U\left(M_{0}+\varepsilon\right)}{d} \varepsilon_{1}-\varepsilon_{2} \quad \forall t \geq s_{q}^{(k)}+T^{*} .
$$

Choose an integer $N_{0}>0$, such that when $k \geq N_{0}$ and $q \geq K^{(k)}$ we have $t_{q}^{(k)}-s_{q}^{(k)} \geq T^{*}+T$. Hence, for any $q \geq K^{(k)}, k \geq N_{0}$ and $t \in\left[t_{q}^{(k)}-T, t_{q}^{(k)}\right]$, we have

$$
\begin{aligned}
\frac{d y\left(t, \varphi_{k}\right)}{d t} & =y\left(t, \varphi_{k}\right)\left[-\left(d+d_{1}\right)+m_{1} U\left(x\left(t, \varphi_{k}\right)\right)\right] \\
& \geq y\left(t, \varphi_{k}\right)\left[-\left(d+d_{1}\right)+m_{1} U\left(x^{*}(t)-\frac{m U\left(M_{0}+\varepsilon\right)}{d} \varepsilon_{1}-\varepsilon_{2}\right)\right] .
\end{aligned}
$$

Integrating (4.42) from $t_{q}^{(k)}-T$ to $t_{q}^{(k)}$, by (4.33) we obtain

$$
\begin{aligned}
\frac{\eta}{k^{2}} & =y\left(t_{q}^{(k)}, \varphi_{k}\right) \\
& \geq y\left(t_{q}^{(k)}-T, \varphi_{k}\right) \exp \int_{t_{q}^{(k)}-T}^{t_{q}^{(k)}}\left[-\left(d+d_{1}\right)+m p\left(x^{*}(t)-\frac{p\left(M_{0}+\varepsilon\right)}{d} \varepsilon_{1}-\varepsilon_{2}\right)\right] d t \\
& \geq \frac{\eta}{k^{2}} \exp \left(\varepsilon_{2}\right) \\
& >\frac{\eta}{k^{2}}
\end{aligned}
$$

which is contradictory. This contradiction shows that there exists a constant $m_{2}>0$ such that

$$
\liminf _{t \rightarrow \infty} y(t) \geq m_{2}
$$

for any positive solution $(x(t), y(t), z(t))$ of system (2.3). Choose $\varepsilon_{3}>0$ such that $m_{2}-\varepsilon_{3}>0$, then there exists a $T_{3}>0$ such that $y(t) \geq m_{2}-\varepsilon_{3}$ for all $t>T_{3}$. From the third equation of system (2.3), we have

$$
\dot{z}(t) \geq-a z(t)+\left(m_{2}-\varepsilon_{3}\right), \quad \forall t>T_{3} .
$$


By the comparison theorem it follows that $z(t) \geq \bar{z}(t)$, for all $t>T_{3}$, where $\bar{z}(t)$ is the solution of the following system with initial condition $\bar{z}\left(T_{3}\right)=z\left(T_{3}\right)>0$ :

$$
\dot{u}(t)=-a u(t)+\left(m_{2}-\varepsilon_{3}\right) .
$$

By simple calculation we have

$$
\bar{z}(t) \geq \frac{m_{2}-\varepsilon_{3}}{a}, \quad \forall t>T_{3} .
$$

Hence, we further obtain

$$
\liminf _{t \rightarrow \infty} z(t) \geq \frac{m_{2}-\varepsilon_{3}}{a}
$$

Let

$$
\underline{m}=\min \left\{\frac{p \exp \left(-\left(d+m_{1}\left(M_{0}+\varepsilon\right) M_{1}\right) T\right)}{1-\exp \left(-\left(d+m_{1}\left(M_{0}+\varepsilon\right) M_{1}\right) T\right)}, m_{2}, \frac{m_{2}-\varepsilon_{3}}{a}\right\} ;
$$

then we have

$$
\liminf _{t \rightarrow \infty} x(t) \geq \underline{m}, \quad \liminf _{t \rightarrow \infty} y(t) \geq \underline{m}, \quad \liminf _{t \rightarrow \infty} z(t) \geq \underline{m} .
$$

This completes the proof of Theorem 4.2.

Returning to the original system (1.4), as a consequence of Theorems 4.1 and 4.2 we have the following corollary.

Corollary 4.3. Suppose that inequality (3.1) holds. Then,

(a) solution $\left(x^{*}(t), 0\right)$ of system (1.4) is globally attractive if and only if

$$
\int_{0}^{T}\left[-\left(d+d_{1}\right)+m_{1} U\left(x^{*}(t)\right)\right] d t \leq 0
$$

(b) system (1.4) is permanent if and only if

$$
\int_{0}^{T}\left[-\left(d+d_{1}\right)+m_{1} U\left(s^{*}(t)\right)\right] d t>0
$$

Remark 4.4. From [18, Theorem 3.2], we find that the results on permanence is similar between model with delayed nutrient recycling and the model with instantaneous nutrient recycling. 
Remark 4.5. According to Corollary 4.3, the sufficient and necessary conditions on the permanence and extinction of the microorganisms are obtained for a chemostat models with delayed nutrient recycling and periodically pulsed input. Obviously, it is an very good extension of the corresponding results given in [18].

\section{Global Attractivity}

Now, we discuss the global attractivity of all positive solutions of system (2.3), we have the following result.

Theorem 5.1. Suppose that inequalities (3.1) and (4.15) hold. If

$$
\alpha d_{1}-\beta d<0,
$$

where $\alpha=\sup _{\theta \in\left[0, M_{0}\right]} U^{\prime}(\theta)$ and $\beta=\inf _{\theta \in\left[0, M_{0}\right]} U^{\prime}(\theta)$, then for any two positive solutions $\left(x_{1}(t), y_{1}(t), z_{1}(t)\right)$ and $\left(x_{2}(t), y_{2}(t), z_{2}(t)\right)$ of system $(2.3)$,

$$
\begin{gathered}
\lim _{t \rightarrow \infty}\left(x_{1}(t)-x_{2}(t)\right)=0, \quad \lim _{t \rightarrow \infty}\left(y_{1}(t)-y_{2}(t)\right)=0, \\
\lim _{t \rightarrow \infty}\left(z_{1}(t)-z_{2}(t)\right)=0 .
\end{gathered}
$$

Proof. From inequality (3.1) and (5.1), there exist two constants $c>0$ and $c_{1}>0$ such that

$$
\begin{aligned}
& b d_{1}+c d-a c<0, \quad a c-\frac{m d_{1}}{m_{1}}<0, \\
& d-c_{1} m_{1} \alpha>0, \quad c_{1} m \beta-\frac{m d_{1}}{m_{1}}>0 .
\end{aligned}
$$

Further, we can choose a constant $\varepsilon_{0}>0$ such that

$$
d-c_{1} m_{1} \alpha_{\varepsilon_{0}}>0, \quad \frac{\underline{m}-\varepsilon_{0}}{c_{1}}\left(c_{1} m \beta_{\varepsilon_{0}}-\frac{m d_{1}}{m_{1}}\right)>0,
$$

where $\alpha_{\varepsilon_{0}}=\sup _{\theta \in\left[0, M_{0}+\varepsilon_{0}\right]} U^{\prime}(\theta)$ and $\beta_{\varepsilon_{0}}=\inf _{\theta \in\left[0, M_{0}+\varepsilon_{0}\right]} U^{\prime}(\theta)$.

Let

$$
V(t)=x(t)+\frac{m}{m_{1}} y(t)+c z(t),
$$


then system (2.3) is equivalent to the following system:

$$
\begin{aligned}
& \frac{d V(t)}{d t}=-d V(t)+\left(a c-\frac{m d_{1}}{m_{1}}\right) y(t)+\left(b d_{1}+d c-a c\right) z(t), \\
& \frac{d y(t)}{d t}=y(t)\left[-\left(d+d_{1}\right)+m_{1} U\left(V(t)-\frac{m}{m_{1}} y(t)-c z(t)\right)\right], \quad t \neq n T, \\
& \frac{d z(t)}{d t}=a y(t)-a z(t), \\
& V\left(t^{+}\right)=V(t)+p, \quad t=n T, n \in N . \\
& y\left(t^{+}\right)=y(t), \\
& z\left(t^{+}\right)=z(t),
\end{aligned}
$$

Let $\left(x_{1}(t), y_{1}(t), z_{1}(t)\right)$ and $\left(x_{2}(t), y_{2}(t), z_{2}(t)\right)$ be any two positive solutions of system (2.3); from Theorems 3.1 and 4.1 we have

$$
\begin{gathered}
\underline{m} \leq \liminf _{t \rightarrow \infty} x_{i}(t) \leq \limsup _{t \rightarrow \infty} x_{i}(t) \leq M_{0}, \\
\underline{m} \leq \liminf _{t \rightarrow \infty} y_{i}(t) \leq \limsup _{t \rightarrow \infty} y_{i}(t) \leq \frac{m_{1}}{m} M_{0}, \\
\underline{m} \leq \liminf _{t \rightarrow \infty} z_{i}(t) \leq \limsup _{t \rightarrow \infty} z_{i}(t) \leq \frac{(a-d) M_{0}}{b d_{1}}
\end{gathered}
$$

for $i=1,2$. Hence, there exists a $T>0$ such that

$$
\begin{gathered}
\underline{m}-\varepsilon_{0} \leq x_{i}(t) \leq M_{0}+\varepsilon_{0} \\
\underline{m}-\varepsilon_{0} \leq y_{i}(t) \leq \frac{m_{1}}{m} M_{0}+\varepsilon_{0} \\
\underline{m}-\varepsilon_{0} \leq z_{i}(t) \leq \frac{(a-d) M_{0}}{b d_{1}}+\varepsilon_{0}
\end{gathered}
$$

for all $t \geq T$. From above inequalities and the theorem of mean value, we can obtain

$$
\begin{gathered}
\left|y_{1}(t)-y_{2}(t)\right| \geq\left(\underline{m}-\varepsilon_{0}\right)\left|\ln y_{1}(t)-\ln y_{2}(t)\right|, \\
U\left(x_{1}(t)\right)-U\left(x_{2}(t)\right)=\frac{d U(\xi(t))}{d s}\left(x_{1}(t)-x_{2}(t)\right),
\end{gathered}
$$

for all $t \geq T$, where $\xi(t)$ is situated between $x_{1}(t)$ and $x_{2}(t)$.

Let

$$
V_{i}(t)=x_{i}(t)+\frac{m}{m_{1}} y_{i}(t)+c z_{i}(t), \quad i=1,2,
$$


then $\left(V_{i}(t), y_{i}(t), z_{i}(t)\right)$ is the solution of system (5.6). Define the Liapunov function as follows:

$$
W(t)=\left|V_{1}(t)-V_{2}(t)\right|+c_{1}\left|\ln y_{1}(t)-\ln y_{2}(t)\right|+c\left|z_{1}(t)-z_{2}(t)\right| \text {. }
$$

Calculating the derivative of $W(t)$, from (5.9) we have

$$
\begin{aligned}
\frac{d W(t)}{d t}= & \operatorname{sign}\left(V_{1}(t)-V_{2}(t)\right) \\
& \times\left[-d\left(V_{1}(t)-V_{2}(t)\right)+\left(a c-\frac{m d_{1}}{m_{1}}\right)\left(y_{1}(t)-y_{2}(t)\right)+\left(b d_{1}+d c-a c\right)\left(z_{1}(t)-z_{2}(t)\right)\right] \\
& +c_{1} m_{1} \operatorname{sign}\left(y_{1}(t)-y_{2}(t)\right) \\
& \times\left[U\left(V_{1}(t)-\frac{m}{m_{1}} y_{1}(t)-c z_{1}(t)\right)-U\left(V_{2}(t)-\frac{m}{m_{1}} y_{2}(t)-c z_{2}(t)\right)\right] \\
& +\operatorname{sign}\left(z_{1}(t)-z_{2}(t)\right)\left[a c\left(y_{1}(t)-y_{2}(t)\right)-a c\left(z_{1}(t)-z_{2}(t)\right)\right] \\
\leq & -d\left|V_{1}(t)-V_{2}(t)\right|+\left(\frac{m d_{1}}{m_{1}}-a c\right)\left|y_{1}(t)-y_{2}(t)\right| \\
& +\left(a c-b d_{1}-d c\right)\left|z_{1}(t)-z_{2}(t)\right| \\
& +c_{1} m_{1} \operatorname{sign}\left(y_{1}(t)-y_{2}(t)\right) \frac{d U(\xi(t))}{d s} \\
& \times\left[\left(V_{1}(t)-V_{2}(t)\right)-\frac{m}{m_{1}}\left(y_{1}(t)-y_{2}(t)\right)-c\left(z_{1}(t)-z_{2}(t)\right)\right] \\
& +a c\left|y_{1}(t)-y_{2}(t)\right|-a c\left|z_{1}(t)-z_{2}(t)\right| \\
\leq & -\left(d-c_{1} m_{1} \alpha_{\varepsilon_{0}}\right)\left|V_{1}(t)-V_{2}(t)\right| \\
& -\frac{\underline{m}-\varepsilon_{0}}{c_{1}}\left(c_{1} m \beta_{\varepsilon_{0}}-\frac{m d_{1}}{m_{1}}\right) c_{1}\left|\ln y_{1}(t)-\ln y_{2}(t)\right| \\
& -\left(d-c_{1} m_{1} \alpha_{\varepsilon_{0}}+\frac{b d_{1}}{c}\right) c\left|z_{1}(t)-z_{2}(t)\right| \\
& -\rho W(t), \\
& (5)
\end{aligned}
$$

where

$$
\rho=\min \left\{d-c_{1} m_{1} \alpha_{\varepsilon_{0}}, \frac{\underline{m}-\varepsilon_{0}}{c_{1}}\left(c_{1} m \beta_{\varepsilon_{0}}-\frac{m d_{1}}{m_{1}}\right)\right\}
$$

From (5.4), we obtain $\rho>0$. On the other hand, we directly obtain

$$
W\left(t^{+}\right)=W(t), \quad \forall t=n T, n \in N .
$$


Hence, for any $t>T$ we have

$$
W(t) \leq W(T) \exp (-\rho t) .
$$

Consequently, $\lim _{t \rightarrow \infty} W(t)=0$. From this, we finally obtain

$$
\begin{gathered}
\lim _{t \rightarrow \infty}\left(x_{1}(t)-x_{2}(t)\right)=0, \quad \lim _{t \rightarrow \infty}\left(y_{1}(t)-y_{2}(t)\right)=0, \\
\lim _{t \rightarrow \infty}\left(z_{1}(t)-z_{2}(t)\right)=0 .
\end{gathered}
$$

This completes the proof of Theorem 5.1 .

As a direct consequence of Theorem 5.1, we have the following corollary on global attractivity of all positive solutions for the original system (1.4).

Corollary 5.2. Suppose that all the conditions of Theorem 5.1 hold. Then for any two positive solutions $\left(x_{1}(t), y_{1}(t)\right)$ and $\left(x_{2}(t), y_{2}(t)\right)$ of system (1.4),

$$
\lim _{t \rightarrow \infty}\left(x_{1}(t)-x_{2}(t)\right)=0, \quad \lim _{t \rightarrow \infty}\left(y_{1}(t)-y_{2}(t)\right)=0 .
$$

\section{Numerical Examples}

In this section, we will give an example to demonstrate the effectiveness of our main results. Consider system (1.4) with the following parameters:

$$
\begin{aligned}
& U(x)=12 \frac{x \exp (-0.2 x)}{0.5+x}, \quad F(t)=2 e^{-2 t}, \quad d=0.5, \quad d_{1}=0.2 \\
& m=0.9, \quad m_{1}=0.8, \quad b=0.4, \quad p=0.5, \quad T=1, \quad a=2 .
\end{aligned}
$$

By calculating, we obtain

$$
\begin{gathered}
\frac{b}{a-d}=\frac{4}{15}, \quad \frac{m}{a m_{1}}=\frac{9}{8}, \\
\int_{0}^{1}\left[-\left(d+d_{1}\right)+m_{1} U\left(x^{*}(t)\right)\right] d t=0.1351>0, \\
x^{*}(t)=\frac{0.5 e^{-0.5(t-n)}}{1-e^{-0.5}}, \quad t \in(n, n+1], n \in N, \\
\alpha d_{1}-\beta d=2.4947>0 .
\end{gathered}
$$

Therefore, inequalities (4.15) hold, but (5.1) does not hold. From Theorem 4.1, we obtain that system (1.4) is permanent. But, from the numerical simulation (see Figures 1 and 2 ) we see that there exists a unique positive $T$-periodic solution $\left(x^{*}(t), y^{*}(t)\right)$ of system (1.4) such that any solution $(x(t), y(t))$ of system (1.4) with initial value $\left(x_{0}, \varphi(\theta)\right)$ tends to $\left(x^{*}(t), y^{*}(t)\right)$ as 


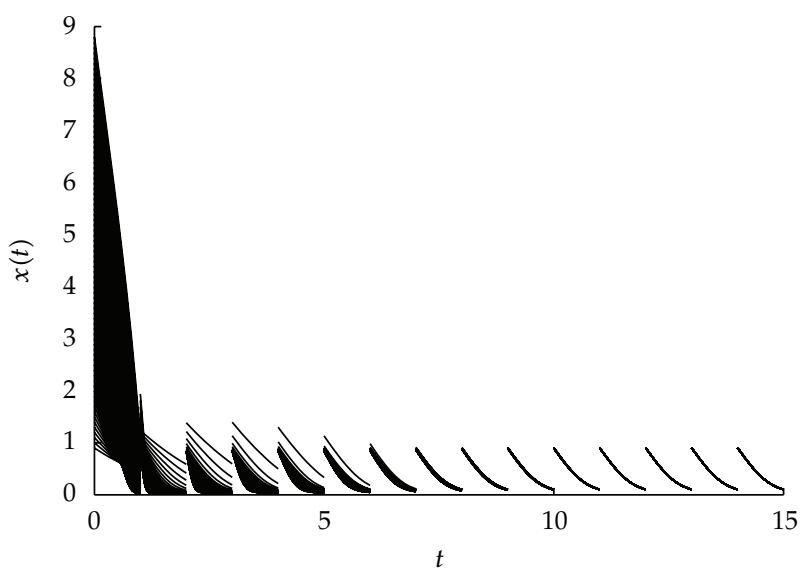

Figure 1: Time series of $x(t)$.

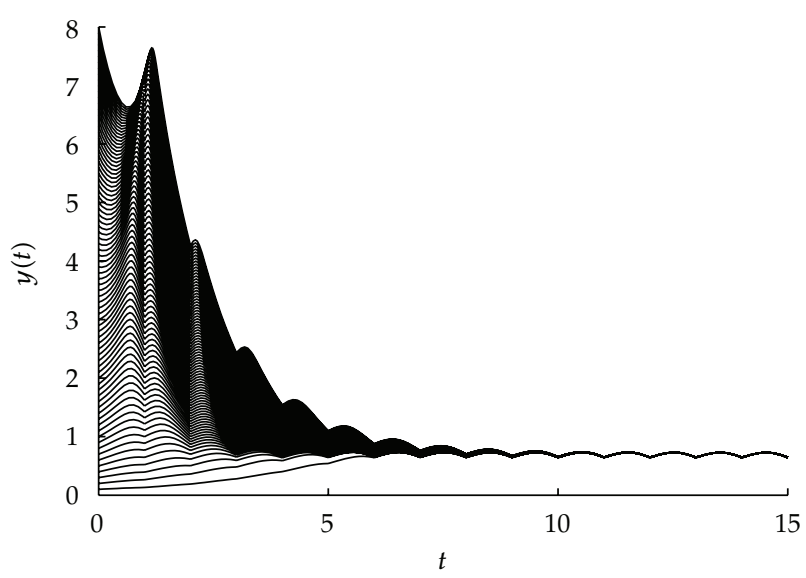

Figure 2: Time series of $y(t)$.

$t \rightarrow \infty$. Therefore, we can guess that if only inequality (4.15) holds then system (1.4) has a unique positive $T$-periodic solution which is globally attractive.

Open Problem. From the above numerical simulation, it prompts us to be able to put forward the following important and interesting open problem, that is, when inequality (3.1) holds then system (1.4) is also globally attractive as long as it is permanent.

\section{Acknowledgment}

This paper was supported by The National Natural Science Foundation of China [10961022, 10901130,11071283], The Scientific Research Programmes of Colleges in Xinjiang [XJEDU2007G01, XJEDU2006I05, XJEDU2008S10, XJEDU2009S21], and the Sciences Foundation of Shanxi [2009011005-3]. 


\section{References}

[1] E. Beretta, G. I. Bischi, and F. Solimano, "Stability in chemostat equations with delayed nutrient recycling," Journal of Mathematical Biology, vol. 28, no. 1, pp. 99-111, 1990.

[2] E. Beretta and Y. Takeuchi, "Global stability for chemostat equations with delayed nutrient recycling," Nonlinear World, vol. 1, no. 3, pp. 191-306, 1994.

[3] G. I. Bischi, "Effects of time lage on transient characteristics of a nutrient cycling model," Mathematical Biosciences, vol. 102, pp. 151-175, 1992.

[4] H. I. Freedman and Y. T. Xu, "Models of competition in the chemostat with instantaneous and delayed nutrient recycling," Journal of Mathematical Biology, vol. 31, no. 5, pp. 513-527, 1993.

[5] M. Gao, H. Shi, and Z. Li, "A planktonic resource-consumer model with a temporal delay in nutrient recycling," Journal of Mathematical Analysis and Applications, vol. 339, no. 1, pp. 511-516, 2008.

[6] Z. Lu and K. P. Hadeler, "Model of plasmid-bearing, plasmid-free competition in the chemostat with nutrient recycling and an inhibitor," Mathematical Biosciences, vol. 148, no. 2, pp. 147-159, 1998.

[7] S. Nakaoka and Y. Takeuchi, "Competition in chemostat-type equations with two habitats," Mathematical Biosciences, vol. 201, no. 1-2, pp. 157-171, 2006.

[8] S. G. Ruan, "Persistence and coexistence in zooplankton-phytoplankton-nutrient models with instantaneous nutrient recycling," Journal of Mathematical Biology, vol. 31, no. 6, pp. 633-654, 1993.

[9] S. Ruan, "Oscillations in plankton models with nutrient recycling," Journal of Theoretical Biology, vol. 208, no. 1, pp. 15-26, 2001.

[10] S. Ruan and X.-Z. He, "Global stability in chemostat-type competition models with nutrient recycling," SIAM Journal on Applied Mathematics, vol. 58, no. 1, pp. 170-192, 1998.

[11] H. L. Smith and P. Waltman, The Theory of the Chemostat, vol. 13 of Cambridge Studies in Mathematical Biology, Cambridge University Press, Cambridge, UK, 1995.

[12] V. S. H. Rao and P. R. S. Rao, "Global stability in chemostat models involving time delays and wall growth," Nonlinear Analysis, vol. 5, no. 1, pp. 141-158, 2004.

[13] V. S. H. Rao and P. R. S. Rao, "Oscillations induced by the time lags in limited nutrient-consumer dynamic models," Chaos, Solitons and Fractals, vol. 25, no. 1, pp. 11-23, 2005.

[14] V. S. H. Rao and P. R. S. Rao, "Mathematical models and stabilizing bio-control mechanisms for microbial populations in a cultured environment," Chaos, Solitons and Fractals, vol. 28, no. 5, pp. 12221251, 2006.

[15] S. Yuan, D. Xiao, and M. Han, "Competition between plasmid-bearing and plasmid-free organisms in a chemostat with nutrient recycling and an inhibitor," Mathematical Biosciences, vol. 202, no. 1, pp. $1-28,2006$.

[16] X.-Z. Meng, Q.-L. Zhao, and L.-S. Chen, "Global qualitative analysis of new monod type chemostat model with delayed growth response and pulsed input in polluted environment," Applied Mathematics and Mechanics, vol. 29, no. 1, pp. 75-87, 2008.

[17] S. Sun and L. Chen, "Dynamic behaviors of Monod type chemostat model with impulsive perturbation on the nutrient concentration," Journal of Mathematical Chemistry, vol. 42, no. 4, pp. 837847, 2007.

[18] Z. Teng, R. Gao, M. Rehim, and K. Wang, "Global behaviors of Monod type chemostat model with nutrient recycling and impulsive input," Journal of Mathematical Chemistry, vol. 47, no. 1, pp. 276-294, 2010.

[19] F. Wang and G. Pang, "Competition in a chemostat with Beddington-DeAngelis growth rates and periodic pulsed nutrient," Journal of Mathematical Chemistry, vol. 44, no. 3, pp. 691-710, 2008.

[20] S. Zhang and D. Tan, "Study of a chemostat model with Beddington-DeAngelis functional response and pulsed input and washout at different times," Journal of Mathematical Chemistry, vol. 44, no. 1, pp. 217-227, 2008.

[21] Z. Zhao and L. Chen, "Dynamic analysis of lactic acid fermentation in membrane bioreactor," Journal of Theoretical Biology, vol. 257, no. 2, pp. 270-278, 2009.

[22] Z. Zhao, L. Chen, and X. Song, "Extinction and permanence of chemostat model with pulsed input in a polluted environment," Communications in Nonlinear Science and Numerical Simulation, vol. 14, no. 4, pp. 1737-1745, 2009. 


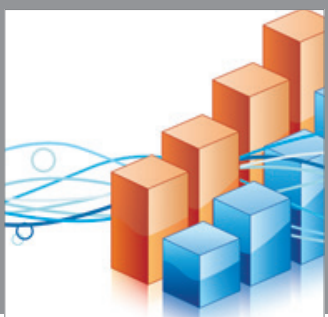

Advances in

Operations Research

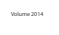

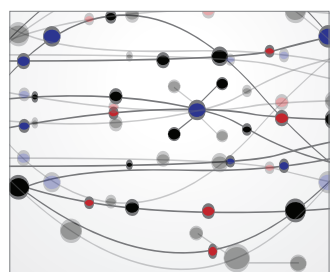

\section{The Scientific} World Journal
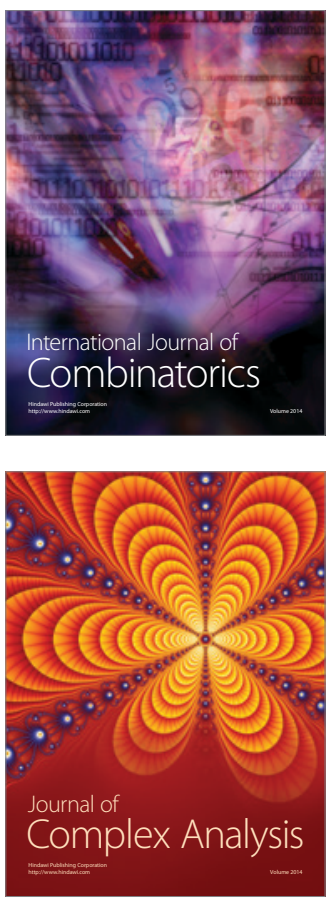

International Journal of

Mathematics and

Mathematical

Sciences
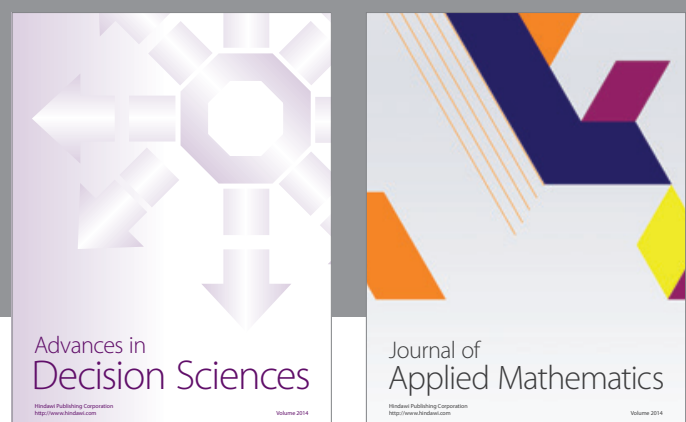

Journal of

Applied Mathematics
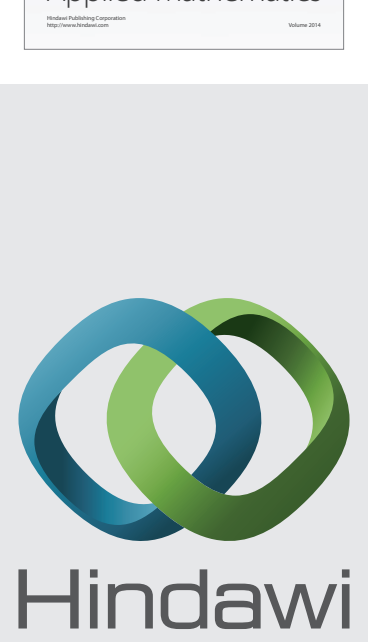

Submit your manuscripts at http://www.hindawi.com
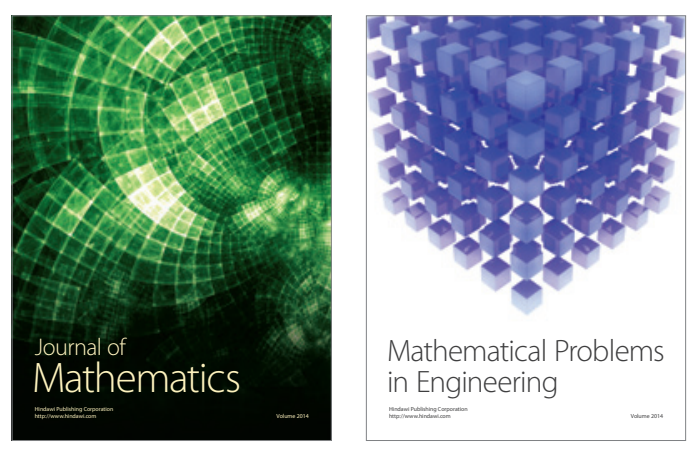

Mathematical Problems in Engineering
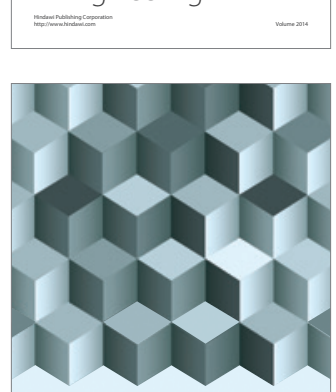

Journal of

Function Spaces
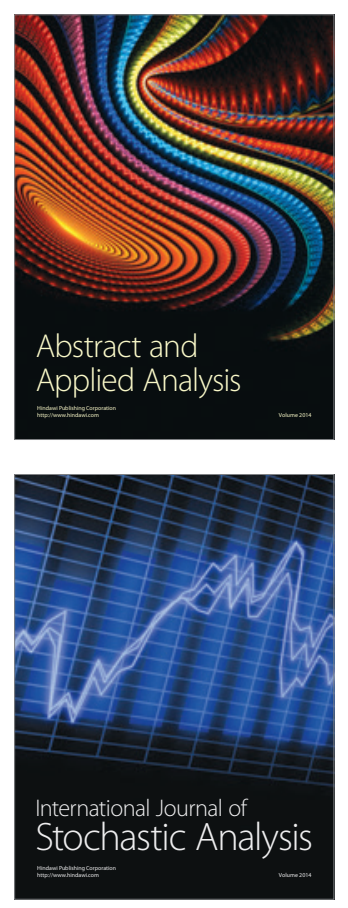

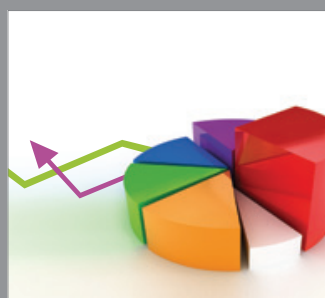

ournal of

Probability and Statistics

Promensencen
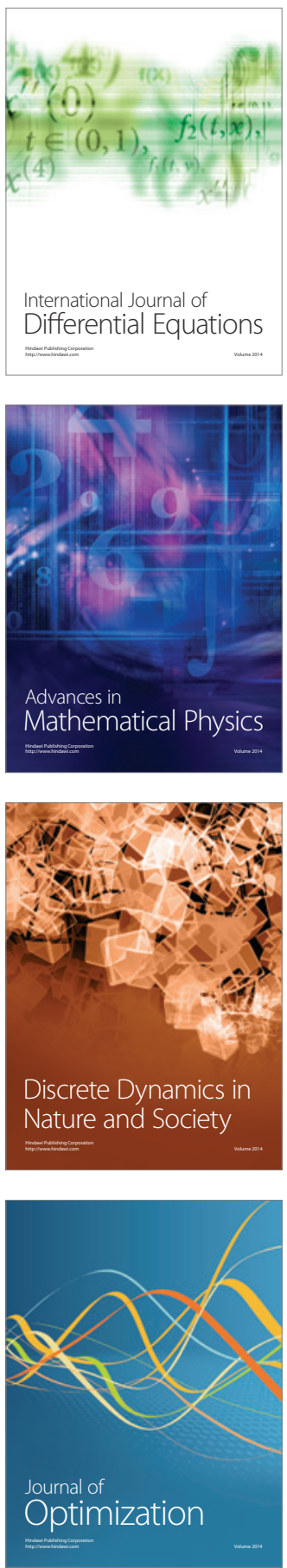